\title{
Belgeo
}

Revue belge de géographie

\section{Le vote blanc et nul en Wallonie : analyse écologique et individuelle}

Blank and null votes in Wallonia: an ecological and individual analysis

\section{Geoffrey Pion}

\section{(2) OpenEdition}

1 Journals

\section{Édition électronique}

URL : http://journals.openedition.org/belgeo/6441

DOI : 10.4000/belgeo.6441

ISSN : 2294-9135

Éditeur :

National Committee of Geography of Belgium, Société Royale Belge de Géographie

\section{Édition imprimée}

Date de publication : 1 octobre 2010

Pagination : 249-264

ISSN : 1377-2368

\section{Référence électronique}

Geoffrey Pion, « Le vote blanc et nul en Wallonie : analyse écologique et individuelle », Belgeo [En ligne], 3 | 2010, mis en ligne le 15 décembre 2012, consulté le 04 mai 2019. URL : http:// journals.openedition.org/belgeo/6441; DOI : 10.4000/belgeo.6441

Ce document a été généré automatiquement le 4 mai 2019.

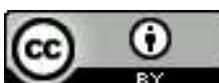

Belgeo est mis à disposition selon les termes de la licence Creative Commons Attribution 4.0 International. 


\title{
Le vote blanc et nul en Wallonie : analyse écologique et individuelle
}

\author{
Blank and null votes in Wallonia: an ecological and individual analysis
}

Geoffrey Pion

\section{Introduction}

1 Malgré la double page traditionnelle du quotidien "Le Soir" à son sujet ces dernières années après chaque élection, le vote blanc et nul est un comportement de vote méconnu. Considéré par certains comme un résidu logique de tout vote, ou confondu avec l'abstention électorale, le vote blanc et nul est resté très peu étudié jusqu'ici aussi bien dans les études électorales françaises qu'anglo-saxonnes. La part qu'il représente est, il est vrai, souvent très faible ( $4 \%$ en moyenne des votes exprimés en France, $2 \%$ en Allemagne, ou encore $0,5 \%$ aux Pays Bas). En Belgique par contre, le scrutin proportionnel et l'obligation de vote, propice comme on le verra à l'expression de ce type de vote, auraient dû pousser à des études sur le phénomène. Nous n'avons pourtant trouvé que peu de traces, dans la littérature belge francophone et anglophone, d'études spécifiques sur ce comportement de vote. Quand des travaux en ont fait état, c'était couplé avec l'abstention électorale (le fait de ne pas aller voter) pour former un "non vote", en des termes plus précis, des votes non valables, ceux qui ne rentrent pas en compte pour la répartition des sièges (Delwit, 1992; De Winter \& Ackaert, 1994; De Winter et al., 2003). Le vote étant obligatoire en Belgique, il est courant de coupler et de confondre les deux, et en règle générale de les sous-estimer largement. Pourtant, aux élections fédérales 2010 c'est plus d'1/4 des Wallons et des Bruxellois qui se sont abstenus ou ont voté blanc et nul contre seulement $15 \%$ en Flandre (Pion \& Van Hamme, 2011).

Deux raisons peuvent expliquer cette méconnaissance d'un vote émis pourtant par plus d'électeurs que par le parti Ecolo hors périodes fastes ou par le FN. D'une part, la dénomination "blanc et nul" est intrigante et peu claire, d'autant plus qu'on les appelle aussi votes annulés, blancs ou non valables ${ }^{1}$. Nous utiliserons dans cet article le terme de vote ou d'électeur "blanc et nul". La façon la plus courante de décrire ces bulletins est la 
suivante : le vote blanc concerne les bulletins vierges de toute inscription, les bulletins nuls sont ceux contraires à la règle électorale, donc annulés. Il peut dans ce cas s'agir d'un bulletin raturé, déchiré, sali, rempli avec un stylo non approprié. Ces bulletins annulés peuvent être le fait d'une volonté de l'électeur ou d'une erreur de vote. Le cas commun est en Belgique de cocher les cases de candidats dans plusieurs listes différentes ou de mal noircir les cases relatives au choix effectué. Ceci est valable en Belgique dans les cantons où se pratique le vote manuel. Dans ceux où le vote électronique a été instauré ${ }^{2}$ il n'est $a$ priori pas possible de voter "nul", on ne peut que choisir de voter blanc. Dans la plupart des démocraties occidentales ${ }^{3}$, les bulletins blancs et nuls sont comptabilisés ensemble, et aucune statistique n'est fournie sur la part de l'un et de l'autre.

D'autre part, les enquêtes par sondages se penchent rarement sur ce comportement de vote. Dans le cas des enquêtes universitaires, le vote blanc et nul est une réponse possible ${ }^{4}$ , mais les résultats sont rarement traités par après. L'argument couramment avancé est qu'il est difficile d'obtenir des échantillons suffisants pour traiter de ce vote, d'autant plus qu'il est moins fréquemment déclaré par les enquêtés par rapport à la réalité. Cette sous-estimation est d'ailleurs peut-être liée au fait que, dans le cas d'une erreur de vote, l'électeur ne se rend pas compte que son vote a été annulé. Dans les enquêtes organisées par les médias, on n'en fait généralement même pas mention.

Après avoir réalisé un tour de la littérature sur les votes blancs et nuls, on abordera l'étude empirique de ce comportement de vote en Région wallonne. Nous tenterons d'abord de montrer sa spatialité ainsi que son évolution depuis les années 1990 . Nous chercherons ensuite à bâtir un modèle afin d'expliquer la variation spatiale du vote blanc et nul en Wallonie. Enfin, nous tenterons d'expliquer les motivations propres à ce vote grâce à l'analyse de plus de 2000 bulletins de vote blancs et nuls recueillis dans six cantons wallons. Ces données originales nous permettront de proposer une typologie des votes blancs et nuls, d'analyser les thèmes et champs lexicaux repris dans les écrits relevés sur ces bulletins et de finalement conclure sur le sens à donner à ces votes.

\section{Etat de l'art}

L'opposition entre votes blancs et nuls est généralement faite pour renvoyer à la dichotomie plus essentielle réalisée entre choix et erreur. On a parfois l'impression que le terme "blanc" aurait une connotation positive, ayant trait à un choix réfléchi et critique, alors que celui de "nul" serait assimilé à l'indifférence et à l'incapacité à exprimer un vote valable. Ainsi Durand (1999) et son association pour la reconnaissance du vote blanc réclament en France que les votes blancs et nuls soient comptabilisés dans les votes valables dans le but d'établir un véritable thermomètre du mécontentement des électeurs. Il avance que la majorité des votes blancs et nuls sont en réalité des votes blancs et qu'ils ont une signification essentielle que la classe politique ne doit pas mettre de côté. Il a été relayé en cela par certains députés et sénateurs de droite comme de gauche, qui ont fait plusieurs propositions de loi en ce sens, sans aboutir jusqu'à présent. A notre connaissance, une seule étude a permis, en France, de distinguer "le bon grain de l'ivraie". Déloye et Ihl (1991) ont ainsi montré que lors des élections législatives de 1881, qui, il est vrai, datent d'avant l'instauration du suffrage universel, seuls $4,3 \%$ des bulletins annulés étaient en réalité des erreurs de vote. En Belgique, Delwit restait pour sa part dans l'expectative au début des années 1990 : “En Belgique, si nous pouvons croire que, parmi les votants, il y a des actes conscients et une réelle volonté de s'abstenir, que 
ce soit à travers la non-participation ou l'expression d'un vote nul ou blanc, il n'y a pas moyen de discerner ces abstentionnistes des personnes mal enregistrées, distraites, malades..." (Delwit, 1992, p. 185). Dès lors, le débat est ouvert sur le sens à donner à ces bulletins de vote.

6 Les études traitant des votes blancs et nuls sont donc rares dans les littératures francophone et anglo-saxonne. Selon nous, quatre modèles explicatifs ressortent tout de même de ces études : deux d'ordre politique (le système politique et la configuration politique de l'élection) et deux d'ordre socio-spatial (le contexte local et familial et le niveau socio-économique).

7 Le système politique, et plus particulièrement l'obligation de vote, est présenté comme la variable la plus robuste d'explication de l'importance des votes blancs et nuls (Uggla, 2008). Ainsi le vote tend à être plus important dans les démocraties de vote obligatoire, particulièrement quand les sanctions prévues pour les abstentionnistes sont effectives. Il n'est ainsi pas étonnant que les rares études réalisées sur le sujet l'aient été dans ce type de pays. Forcés de voter sous peine de dures sanctions administratives, les électeurs australiens, argentins ou brésiliens sont plus enclins à émettre des votes blancs et nuls que les électeurs français ou américains qui n'ont aucune obligation de se rendre au bureau de vote. Le système électoral joue aussi un rôle important sur la participation. Ainsi un scrutin de type majoritaire crée des conditions favorables à l'émission de votes blancs et nuls lors des seconds tours où le choix se fait entre 2 voire 3 candidats, parfois suite à une consigne de vote (Bon \& Cheylan, 1988). Zulfikarpasic (2001) constate que le niveau de votes blancs et nuls est systématiquement supérieur au second tour des élections présidentielles françaises. Bussi note à ce propos que la carte du vote blanc et nul au second tour des élections présidentielles est généralement proche de celle du vote Front National, parti qui n'atteint que très rarement le second tour (Bussi, 1998). Enfin, les référendums, qui ne sont pas légalement possible en Belgique, entrainent aussi une forte émission de votes blancs et nuls étant donné le choix binaire proposé.

8 Notre cas d'étude aura donc pour objet un pays où le vote obligatoire crée des conditions propices à l'émission de votes blancs et nuls.

9 Les conditions politiques de l'élection sont aussi un facteur explicatif du vote. En plus de la situation du deuxième tour dans un scrutin majoritaire, il semble que la compétition politique et la prédominance d'un parti politique dans la vie politique nationale ou locale fait augmenter le niveau de votes blancs et nuls. Ainsi, plus le scrutin est serré et indécis, et plus le choix partisan est large, plus les votes blancs et nuls représentent une proportion marginale des votes (Power \& Roberts, 1995 ; Zulfikarpasic, 2001 ; Uggla, 2008). Delwit affirme ainsi pour le cas belge que "si le débat se situe dans un consensus mou ou perçu comme tel, la mobilisation sera d'autant plus faible et l'abstention d'autant plus grande" (Delwit, 1992, p. 80). Mais ce refus de choisir se marque dans une démocratie de vote obligatoire plus fréquemment par un vote blanc et nul que par une abstention, par peur des sanctions. De Winter et al. (2003) avancent ainsi que, logiquement, si le vote obligatoire ne devait plus avoir cours en Belgique, pratiquement la totalité des votants "blancs et nuls" s'abstiendraient. Bussi et al. (2004) montrent pour leur part que la proportion de votes blancs et nuls est maximale en France dans les cantons qui votent soit très à droite, soit très à gauche: "dans un village où un courant politique est hégémonique, ne pas voter, c'est déjà s'afficher non anonymement dans l'opposition : le vote 'blanc' devient alors une forme d'abstention plus discrète". Dans le même ordre d'idée, Delruelle, Vosswinkel et al. (1989) constatent que lors des élections communales 
belges, le vote blanc et nul est maximal dans les petites communes rurales où le choix partisan est souvent binaire. Enfin, il ne semble pas y avoir de lien net entre le type d'élection (européenne, nationale ou locale) et le vote blanc et nul, certains notant un niveau de vote blanc et nul légèrement plus faible aux élections européennes en France "car c'est le scrutin qui présente le moins d'intérêt aux yeux des électeurs" (Zulfikarpasic, 2001, p. 257), quand d'autres constatent l'inverse (Delwit, 1992).

Nous rajoutons ici dans les conditions de l'élection le type de vote, dans le sens manuel ou électronique. A la manière de nombreux pays où les électeurs ont différents choix pour voter (par voie postale, par internet, avec des bureaux itinérants, de façon manuelle ou électronique), la Belgique connaît deux types différents de votes: le manuel et l'électronique. Or, comme nous l'avons précisé, dans l'un (le vote électronique), il n'est en théorie possible que de voter blanc. Cela constitue à notre sens une différence notable et nous inclurons une variable binaire la matérialisant.

11 Au niveau individuel, la thèse de l'incompétence est marquée comme une variable très positivement corrélée aux votes annulés au Brésil (Power \& Roberts, 1995). Dans la plus grande démocratie de vote obligatoire au monde, où les sanctions pour les abstentionnistes sont bien réelles, le niveau d'instruction et notamment l'analphabétisme sont un facteur décisif pour expliquer les votes annulés. En France, le niveau d'instruction est aussi pointé du doigt comme un facteur explicatif du vote blanc et nul mais de façon inverse par rapport au Brésil, étant donné que ce seraient les personnes les plus éduquées et politisées qui émettraient ces votes (Zulfikarpasic, 2001). Cette incompétence serait aussi le fait d'électeurs votant pour la première fois, du fait de leur arrivée à l'âge de voter, 18 ans en Belgique, ou de leur naturalisation récente (McAllister \& Makkai, 1993; Power \& Roberts, 1995). La complexité du scrutin et de la manière d'émettre un vote valable peut aussi faire augmenter les erreurs de vote. Ainsi en Italie, il faut faire son choix par rapport à un sigle, le nom des partis n'étant pas écrit sur les bulletins de vote. Ceci expliquait notamment les confusions entre listes communistes dans les années 1980, qui présentaient toutes une faucille et un marteau, de la part des électeurs italiens vivant en Belgique, ainsi que le taux globalement élevé, tournant autour de $15 \%$ des votes exprimés, de votes blancs et nuls (Pion, 2010).

12 Nous testerons donc dans la partie empirique l'influence du niveau socio-économique, du niveau d'éducation et du contexte familial.

Enfin, le contexte local influence aussi fortement l'émission de ces votes. Ainsi, en Europe, le vote blanc et nul est surtout connu pour être un vote rural et traditionnel, une abstention cachée (Bussi et al., 1994 ; Delruelle-Vosswinkel et al., 1989 ; Steifbold, 1965). Là où le contrôle social et la culture politique sont forts, le vote blanc et nul est souvent une alternative moins voyante que l'abstention pure et simple (Delruelle, Vosswinkel et al., 1989 ; Bussi, 1998). Au Brésil, par contre, les zones amazoniennes combinent haut taux d'abstention et de votes blancs et nuls alors que les métropoles côtières votent surtout blanc et nul (Power \& Roberts, 1995). Enfin, le contexte familial semble aussi jouer en partie sur le vote blanc et nul, De Winter \& Ackaert (1994) ayant montré que le fait d'être isolé est significativement lié au "non vote", leur étude ayant le biais d'associer abstention électorale et votes blancs et nuls.

14 Nous tiendrons donc compte du niveau d'urbanité dans notre étude agrégée et de la localisation spatiale des bulletins blancs et nuls analysés dans celle à l'échelle individuelle ainsi que du fait d'être isolé ou en couple dans son ménage ${ }^{5}$. 


\section{Variables explicatives}

De ce tour de la littérature, nous avons établi une série de variables qui seront testées afin de comprendre la spatialité du vote blanc et nul en Wallonie.

En premier lieu, on testera l'influence du type de vote (manuel ou électronique) sur le vote blanc et nul. L'erreur de vote n'étant pas possible lors d'un vote électronique, nous postulons que le vote blanc et nul ne devrait recouvrir que son aspect blanc dans les cantons où le vote est électronique et ainsi être de moindre intensité6. En d'autres termes, nous postulons que le mode de vote contribue à la variation spatiale du vote blanc et nul en Wallonie étant donné que les électeurs votant électroniquement ne peuvent pas voir leur vote annulé, cela n'étant pas prévu par la procédure de vote.

Ensuite nous tiendrons compte du niveau socio-économique via la première composante d'une analyse en composantes principales reprenant une série de variables socioéconomiques et qui oppose dans le plan factoriel les territoires aisés aux défavorisés ${ }^{7}$. On évaluera aussi l'impact de données démographiques sur base de deux hypothèses :

1. Les primo-votants et les récents naturalisés, ayant une moindre expérience du vote, sont plus enclins à émettre un vote blanc et nul par méconnaissance du jeu partisan ou suite à une erreur de vote, surtout quand ils sont les seuls à pouvoir voter dans le ménage

2. La forte influence frontalière, mesurée par la présence de frontaliers, de ménages belgofrontaliers $^{9}$ et de Belges travaillant de l'autre côté des frontières française et luxembourgeoise qui seraient ainsi moins concernés par la politique belge s'en détourneraient en émettant un vote blanc ou nul.

Afin de tester ces hypothèses, nous corrélerons le vote blanc et nul avec la part d'étrangers naturalisés ${ }^{10}$, la part des actifs belges travaillant à l'étranger et la part des ménages belgo-frontaliers ${ }^{11}$.

De plus, nous testerons l'influence du niveau d'éducation standardisé par l'âge et le sexe avec pour hypothèse que les personnes ayant un faible niveau d'éducation sont plus susceptibles d'émettre un vote erroné.

\section{Géographie du vote blanc et nul en Wallonie (1991-2009)}

Les données agrégées portent sur neuf élections fédérales et européennes entre 1991 et 2009 et l'échelle d'analyse spatiale est le canton, plus petit agrégat pour lequel des données sont disponibles officiellement auprès du Ministère de l'Intérieur ${ }^{12}$. La proportion de votes blancs et nuls sera rapportée au nombre d'inscrits et non pas au nombre de votes exprimés, afin de tenir compte de l'abstention électorale qui oscille selon les cantons entre 4 et $16 \%$ des inscrits. Afin d'obtenir une spatialisation du vote blanc et nul ces vingt dernières années, nous avons eu recours aux champs de forces développés par Bon \& Cheylan (1988). Cet indicateur calculé grâce à une analyse en composantes principales permet d'évaluer l'importance d'un comportement de vote et sa stabilité sur une période donnée (figure 1). Le champ de force équivaut à la part de la variance expliquée par le premier facteur. Dans notre cas, le facteur 1 explique $83,4 \%$ de la variance totale, ce qui marque la grande stabilité spatiale du vote blanc et nul en Wallonie entre 1991 et 2009. Le vote blanc et nul est donc depuis vingt ans un vote essentiellement rural, particulièrement marqué le long des frontières française et 
luxembourgeoise. Il est cependant plus important dans les centres urbains que dans leurs banlieues. Il est minimal dans les cantons périurbains aisés du Brabant Wallon et dans les arrondissements de Liège, Huy et Waremme. Notons qu'au sein de l'espace belge, le taux de votes blancs et nuls est aujourd'hui presque deux fois supérieur en Wallonie par rapport à la Flandre et à Bruxelles. Cela n'a pourtant pas toujours été le cas; dans les années 1950-1960, les pourcentages les plus importants concernaient en effet des cantons ruraux flamands (Pion \& Van Hamme, 2011). On testera aussi nos variables explicatives avec le fond de vote blanc et nul en début (Fédérales 1991) et le plafond en fin de période analysée (Européennes 2009) ${ }^{13}$ afin de mesurer l'évolution de l'influence de nos facteurs explicatifs sur le vote blanc et nul ces vingt dernières années.

Figure 1. Champs de force du vote blanc et nul en Wallonie (1991-2009).

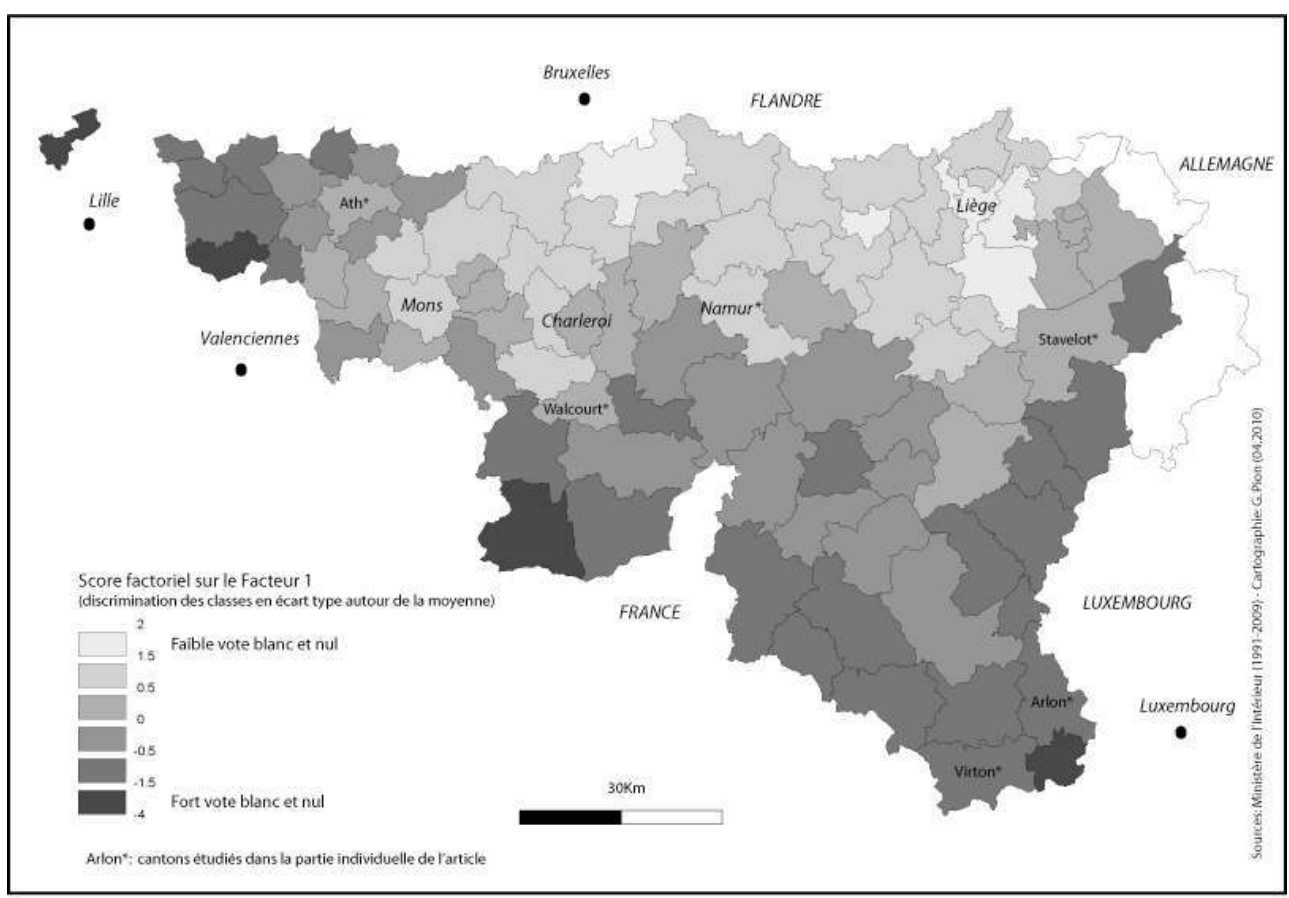

\section{Vers un modèle explicatif du vote blanc et nul en Wallonie au niveau agrégé}

Les études concernant le vote blanc et nul ont donc essentiellement été réalisées au niveau agrégé par le biais des modèles d'explication écologique, ou individuel via des sondages. Notre volonté est ici de coupler une analyse écologique et une analyse individuelle originale, non pas fondée sur une enquête sortie des urnes mais basée sur le dépouillement de plus de 2000 bulletins de vote récupérés dans différents services d'archives électorales.

On a vu que le champ de force du vote blanc et nul permettait de mettre en évidence les zones de forces de ce comportement sur les vingt dernières années. Il convient maintenant de tenter d'expliquer sa répartition spatiale grâce à une régression multiple qui tentera de mettre en lien des indicateurs relatifs au vote blanc et nul (champ de force et résultats bruts) avec les variables explicatives présentées précédemment. 

Trois variables sont très significativement liées au vote blanc et nul : le degré d'urbanité
(le vote blanc et nul augmente quand la densité baisse), la proportion d'actifs travaillant à
l'étranger (le vote blanc et nul augmente quand cette proportion grandit) et la part de frontaliers (évolution identique à la précédente variable). Le second, qui cherche à comprendre la variation spatiale du vote blanc et nul aux européennes 2009, explique $65 \%$ de la variance. Les ménages belgo-frontaliers sont cette fois une variable explicative plus puissante que les Belges travaillant à l'étranger, bien qu'elles restent toutes les deux significatives. Le vote électronique (la part de vote blanc et nul augmente quand le vote tend à être manuel) et le niveau socio-économique deviennent les deux variables les plus significatives pour expliquer le vote blanc et nul. La densité de population perd quant à elle très significativement de son importance alors que la variable "primo-votants" devient significative ${ }^{15}$. Enfin, le troisième et dernier modèle lie champ de force du vote blanc et nul sur la période 1991-2009 et nos variables explicatives. $79 \%$ de la variance est cette fois expliquée. Cinq des sept variables entrent significativement en compte pour ce modèle: le niveau socio-économique, le niveau d'éducation, l'influence frontalière, le travail à l'étranger et le vote électronique. Le niveau d'urbanité et l'importance des primo-votants sont proches d'une significativité au niveau de confiance de 0,1.

Ces modèles marquent donc bien plusieurs phénomènes : 1 . le caractère frontalier du vote blanc et nul ; 2. son aspect rural en début de période et partagé entre urbain et rural en fin ; 3. l'incidence de l'instauration du vote électronique dans certains cantons sur le taux de votes blancs et nuls. Essayons maintenant d'effectuer une analyse individuelle des bulletins de vote afin de la mettre en rapport avec ces premières conclusions.

Tableau 1. Coefficients de régression multiple non standardisés pour le vote blanc et nul en Wallonie.

\begin{tabular}{|l|l|l|l|l|}
\hline Variables & & $\begin{array}{l}\text { B\&N Fédérales } \\
1991\end{array}$ & $\begin{array}{l}\text { B\&N Européennes } \\
2009\end{array}$ & $\begin{array}{l}\text { Champs de force } \\
\text { B\&N (1991-2009) }\end{array}$ \\
\hline $\begin{array}{l}\text { Niveau socio- } \\
\text { économique }\end{array}$ & & 0,1796 & $\mathbf{0 , 3 1 8 4 ^ { * * * }}$ & $\mathbf{0 , 3 2 9 9 ^ { * * * }}$ \\
\hline Urbanité & & $(0,112)$ & $(0,090)$ & $(0,071)$ \\
\hline & $-\mathbf{0 , 3 3 7 6 ^ { * * * }}$ & $-0,0597$ & $-0,1766$ \\
\hline Niveau d'éducation & $(0,127)$ & $(0,129)$ & $(0,101)$ \\
\hline
\end{tabular}




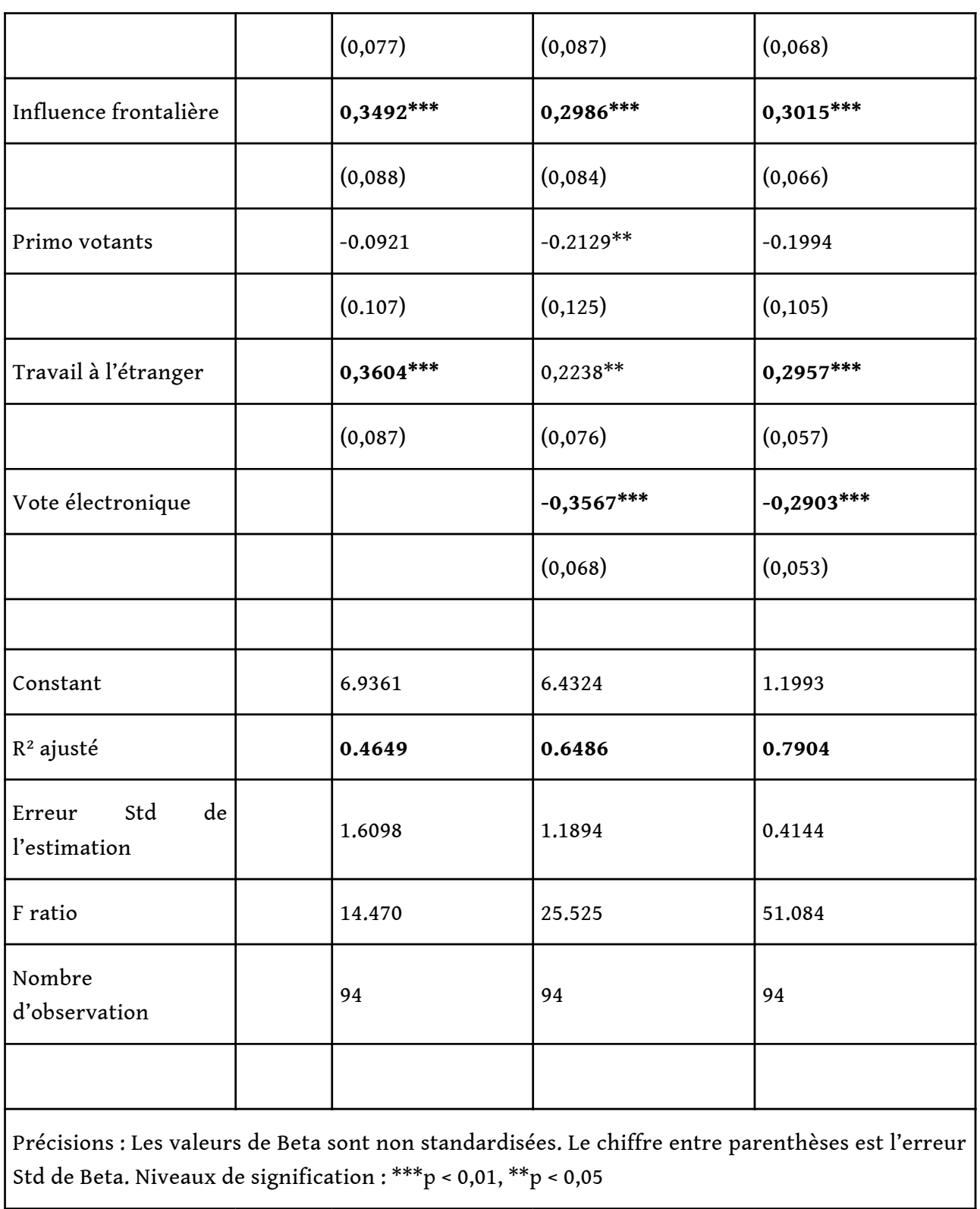

\section{Vers une typologie des votes blancs et nuls : blancs, nuls ou erreur?}

Nous allons maintenant travailler sur une base de données originale provenant de la consultation d'archives électorales. Nous avons récupéré plus de 2000 bulletins de vote blancs et nuls provenant de quatorze bureaux de dépouillement répartis sur six cantons. La volonté est ici de travailler au niveau individuel sur les bulletins blancs et nuls afin de discerner la proportion de votes blancs, non-blancs et d'erreurs de vote d'une part, et d'essayer de donner un sens à ces votes d'autre part.

On a récupéré à peu près autant de bulletins issus du scrutin régional (1060) que du scrutin européen (962) ayant eu lieu tous deux le même jour (le 7 juin 2009). Les deux élections se déroulent le même jour depuis bientôt 15 ans et font passer de fait le scrutin européen au deuxième plan des préoccupations électorales de la part des médias et des 
partis politiques. La Belgique étant un pays fédéral, le scrutin régional y revêt en effet une importance majeure, surtout dans une période de crise institutionnelle et de conflits communautaires comme c'est le cas depuis 2007. Les résultats à l'issue du scrutin régional ont fait apparaître une large percée d'Ecolo au détriment du Parti Socialiste, du Front National et du Mouvement Réformateur. Malgré des prévisions alarmistes, l'abstention électorale n'a que peu augmenté par rapport aux fédérales 2007, alors que le vote blanc et nul a encore gagné du terrain pour atteindre les $9 \%$ des votes exprimés. Les élections européennes ont connu des résultats proches même si le faible nombre de députés à envoyer au Parlement Européen n'a pas changé aussi fondamentalement le rapport de force entre partis qu'à la Région.

Les bulletins de vote proviennent donc de six cantons ayant des caractéristiques variées. Si celui de Namur comprend la ville de Namur, capitale de la Wallonie et troisième métropole de la Région, ceux de Stavelot et Ath sont essentiellement ruraux, agglomérant un petit centre et les campagnes avoisinantes. Le canton de Walcourt agglomère pour sa part une quinzaine d'anciennes communes rurales et semi-rurales situées dans l'aire d'influence de Charleroi. Enfin, les cantons de Virton et d'Arlon sont localisés le long des frontières française et luxembourgeoise et sont fortement marqués par les relations transfrontalières de leur population.

Les résultats de cette étude nous amènent à une typologie détaillée concernant la forme du vote annulé (blanc, nul, erreur) et le sens à lui donnr. Elle va nous permettre d'éclaircir la question suivante : quelle semble être la part d'incompétence, d'indifférence et de protestation parmi les motifs d'émission de ces suffrages?

Le premier résultat, qui paraitt le plus évident dans un premier temps, concerne le partage entre votes blancs et autres votes annulés. On voit dans le tableau 2 que la part de votes blancs représente entre 31 et $49 \%$ des votes annulés. Le maximum est atteint dans les deux cantons ruraux de Ath et Virton, le minimum dans ceux plus urbains de Namur, Arlon et Walcourt. Cependant, les situations divergent entre la proportion de votes blancs et celle de votes blancs et nuls au total. Virton, à la frontière française, est un des cantons où le vote blanc et nul est le plus important de Belgique (près de $12 \%$ des votes), alors qu'à Ath dans la zone rurale du Hainaut occidental, il n'est que de $7 \%$, ce qui en fait l'un des cantons wallons où il est le plus faible, pour ce qui concerne le vote manuel. Si l'on pourrait être tenté d'attribuer la forte proportion de votes blancs et nuls à Virton à la forte proportion de votes vierges de toute trace de vote, la situation du canton d'Ath vient relativiser le constat.

Tableau 2. Caractéristiques des cantons et opposition entre vote blanc et non-blanc (Régionales, Européennes 2009).

\begin{tabular}{|l|l|l|l|l|l|l|}
\hline Cantons & $\begin{array}{l}\text { Nombre de } \\
\text { bulletins }\end{array}$ & Elections & $\begin{array}{l}\% \text { blancs et nuls par } \\
\text { bureau blancs et } \\
\text { dépouillement }\end{array}$ & $\begin{array}{l}\% \text { de } \\
\text { nuls par } \\
\text { canton }\end{array}$ & blancs & $\begin{array}{l}\% \\
\text { autres }\end{array}$ \\
\hline Arlon & 279 & Région & 8,6 & 8 & 33,3 & 66,7 \\
\hline Ath & 84 & Région & 6,5 & 7,3 & 40,5 & 59,5 \\
\hline Namur & 716 & $\begin{array}{l}\text { Région \& } \\
\text { Europe }\end{array}$ & 8 & 7,1 & 31,8 & 68,6 \\
\hline
\end{tabular}




\begin{tabular}{|l|l|l|l|l|l|l|}
\hline Stavelot & 190 & Europe & 9 & 9,3 & 38,9 & 61,1 \\
\hline Virton & 139 & Europe & 11,9 & 12,1 & 48,9 & 51,1 \\
\hline Walcourt & 614 & $\begin{array}{l}\text { Région \& } \\
\text { Europe }\end{array}$ & 8,4 & 8,5 & 32,2 & 67,8 \\
\hline
\end{tabular}

31 Maintenant que nous avons séparé le vote blanc du reste, comment se répartissent les autres types de votes que l'on serait désormais tenté d'appeler nuls ? Il apparaît qu'entre la moitié et les deux tiers des votes non blancs sont des bulletins barrés ou rayés (tableau 3). Ce type de bulletins est particulièrement fréquent dans les bureaux de dépouillement ruraux, à Stavelot, Virton et Ath. Les bulletins contenant des mots, injonctions, phrases, dessins, voire pamphlets, représentent quant à eux entre 14 et $23 \%$ des votes non blancs.

Tableau 3. Types de votes non blancs (Régionales et Européennes 2009).

\begin{tabular}{|l|l|l|l|l|l|l|}
\hline \multirow{2}{*}{ Canton } & \multirow{2}{*}{$\begin{array}{l}\text { Nombre de bureaux de } \\
\text { dépouillement }\end{array}$} & \multicolumn{3}{|l|}{ En \% des votes blancs } \\
\cline { 4 - 7 } & & & \multicolumn{2}{|l|}{ Erreur } & Barrén & Inscription \\
\hline Arlon & 2 & Région & 29,6 & 56,5 & 14,0 \\
Ath & 1 & Région & 16,0 & 68,0 & 16,0 \\
Namur & 5 & Région \& Europe & 29,2 & 47,0 & 23,0 \\
Stavelot & 1 & Europe & 15,5 & 63,8 & 20,7 \\
Virton & 1 & Europe & 16,9 & 69,0 & 22,5 \\
Walcourt & 4 & Région \& Europe & 19,0 & 61,8 & 19,2 \\
\hline
\end{tabular}

32 Cette proportion varie peu selon les bureaux de dépouillement. On peut juste noter que c'est dans la métropole namuroise que les électeurs blancs et nuls tendent le plus à exprimer leur pensée par des mots ou des dessins plutôt que par un silence ou un grand trait.

Nous nous sommes lancé dans une analyse textuelle afin de donner du sens aux bulletins annulés où se trouvent des mots, phrases ou injonctions. Notre première idée était d'utiliser les techniques de statistiques textuelles (Guérin-Pace, 1997), mais deux constats nous ont conduits à ne pas les utiliser malgré leur robustesse. D'une part, la statistique textuelle est le plus souvent utilisée afin de mettre en lumière des réponses à des questions ouvertes lors d'entretiens ou des textes. Or, il ne s'agit ici que de mots ou d'une phrase mais très rarement plus. Ainsi, seuls 6,5\% des bulletins annotés contiennent ne serait-ce qu'une phrase. La plupart des annotations comprises dans ces bulletins de vote blancs et nuls sont davantage des injonctions, sans verbes ni pronoms personnels, ce qui a contribué à notre insatisfaction quant aux premières recherches exploratoires avec ces méthodes. D'autre part, les annotations relevées sur les bulletins se caractérisent par un nombre très important de fautes d'orthographe et de mots illisibles. Il nous est apparu 
ainsi que si nous voulions utiliser des techniques de statistiques textuelles, il nous faudrait reprendre chaque mot, chaque expression afin de corriger l'orthographe et le cas échéant, retirer de l'analyse les phrases ou injonctions comprenant des mots ou expressions inintelligibles. Cette démarche aurait de fait à la fois enlevé du sens aux annotations et une bonne partie du contenu original de l'information empirique.

Nous nous sommes donc tourné vers une analyse plus qualitative à la manière de l'étude de Délhoye \& Ihl sur les bulletins annulés de 1881 dont nous avons déjà parlé. Ils avaient ainsi dichotomisé leurs bulletins entre "refus de choisir" et "explications du choix". Nous verrons que nos bulletins ne suivent pas cette opposition, notamment du fait que l'étude de Délhoye \& Ihl (1991) a été réalisée sur des élections datant du début de l'ère électorale, alors que les électeurs étaient encore en phase d'apprentissage de la règle élémentaire du suffrage universel, à savoir le choix, ni plus ni moins, d'un candidat ou d'un parti sans justification, ni nécessaire ni possible ${ }^{16}$.

$35 \mathrm{Au}$ vu des bulletins recueillis, une distinction fondamentale peut être faite entre les annotations relevant du désintérêt et de l'indifférence, d'une part, et de la protestation, du mécontentement voire de la colère, d'autre part.

Le premier type est composé de personnes ayant annoté sur leur bulletins des mots, phrases, injonctions n'ayant pas de lien avec la politique ou l'élection. Cette expression d'indifférence peut se traduire par des dessins, des injonctions dont on ne perçoit pas le sens comme : "Buzz l'éclair" ou "Jo la fuite" ou relatifs à un événement d'actualité sans lien avec la politique : Standard champion" ou "RSCA Anderlecht" ${ }^{17}$. Ce type d'écritures concerne $24,3 \%$ des bulletins annotés.

Le second type regroupe lui des personnes émettant une protestation ayant trait au champ politique. Ce rejet du monde politique est marqué dans près de deux tiers des bulletins annotés. Cela peut prendre la forme d'une mise en cause du système politique et des hommes politiques, d'une demande particulière ou d'un avis sur le système électoral. Ces sentiments d'électeurs sont dans un tiers des cas émis par le biais d'insultes. Cette protestation, qui semble exprimer une colère par l'utilisation de termes durs et insultants vis-à-vis de la politique, peut s'exprimer par un refus de choisir parmi les choix proposés ou par la proposition, farfelue ou non, d'un autre choix (7,9 \% des cas) : "Sarko - Obama", "pour moi", "Je vote pour ma femme". Elle peut prendre la forme, plus courante, d'une dénonciation tous azimuts du système politique ("Pauvre Belgique - Une politique pour assister"18, "Démocratiquement dégoûté") ou surtout des hommes politiques ("Tous pourris", "Allez à la merde, à quoi sa sert de voté", "en ficelle les crayons et nos dirigeants détournent des millions", "Merde à tous les politiciens. Beaucoup de promesses mais jamais rien de concret"). On y retrouve beaucoup de termes faisant partie du champ lexical de la confiance, de la promesse et de la corruption, toujours pris dans un sens négatif. De plus, les hommes politiques sont souvent tous mis dans le même sac avec l'utilisation de termes de généralisation (tous, bande...) qui revient dans $14,6 \%$ des annotations ou de termes relatifs à la deuxième personne du pluriel (allez, vous...) dans $9 \%$ des bulletins. Quelques bulletins émettent aussi un avis sur le vote obligatoire: "Je suis contre le vote obligatoire", "DROIT $\neq$ OBLIGATION" mais cela reste marginal ( $2 \%$ des annotations). Au final, toutes ces récriminations envers la politique pourraient être résumées par ce bulletin comprenant la justification la plus étayée que nous ayons recueillie : "Voter à quoi ça sert quand on constate que tous ces beaux visages souriants et mis en valeur par un photographe professionnel, ne sont que des mouches engluées dans la marmelade qu'est notre système politique. Promesse encore et toujours, et surtout 
toujours rien, le vide. Alors si mon vote est nul, autant dire ce que je pense et exprimer ainsi tout mon mépris pour ces incompétents. Bcp [Beaucoup] trop nombreux pour une bonne gestion du pays !" (Namur, Régionales 2009).

Enfin un troisième type d'annotation doit être mentionné ici, celles qui ne nous donnent aucune information sur le sens à donner au vote car il est écrit "nul" dessus (5,6\% des cas).

Les bulletins annotés nous permettent donc, au contraire des bulletins blancs et rayés, de donner un sens aux votes annulés. Le mécontentement et la protestation apparaissent donc comme la raison principale de l'émission d'un vote annoté, alors que l'indifférence apparente n'en concerne qu'un quart.

Ces analyses sur les votes blancs, annotés et griffonnés nous amènent à étudier les votes restants, ceux qui ne sont ni un vote blanc ni un vote griffonné ou expliqué, mais marquent une erreur de vote. L'hypothèse que le vote blanc et nul est essentiellement le fait d'incompétence à émettre un vote valable est celle émise par les élites politiques, car la moins dangereuse pour eux (De Winter et al., 2003). Si tel était le cas, le vote blanc et nul ne serait effectivement qu'un résidu normal du vote et ne serait pas porteur d'une opinion de la part des électeurs ayant émis ce vote. Or, il apparait ici que ces erreurs représentent entre 8 et $26 \%$ des votes blancs et nuls émis (tableau 4). Dans un bureau de dépouillement comme le 13e du canton de Namur, cela représente un peu plus de $2 \%$ des votes exprimés. La variation est ici importante entre bureaux de dépouillement, plus que pour les autres types de votes non blancs. La composition même de la population et son niveau de compétence (notamment niveau d'éducation et d'intérêt pour la vie politique) semble être essentiels pour comprendre ce type de votes non valables.

Ces 319 votes blancs et nuls que l'on a déterminés comme étant en réalité des erreurs de vote sont le fait de personnes ayant émis un choix qui a été jugé comme contraire à la loi électorale ${ }^{19}$ : cocher une ou plusieurs cases dans plusieurs listes différentes, rajouter une ou des cases, mal noircir la ou les cases choisies. Dans tous les cas, ces électeurs ont émis un choix partisan, mais aussi souvent au moins un choix de trop, ce qui a amené leur bulletin à être annulé. Nous avons donc relevé les partis choisis par ces électeurs afin d'évaluer les préférences partisanes des électeurs ayant effectué une erreur de vote. On constate ainsi une fragmentation politique marquée, sans parti dominant ce type de votes. Par exemple, à Walcourt, chacun des six courants politiques majeurs (PS, MR, cdH, Ecolo, extrême droite et extrême gauche) attire à lui entre 10 et $20 \%$ des bulletins annulés. Le canton d'Ath, fief socialiste, se caractérise pour sa part par près de 4 bulletins sur 10 annulés comprenant un choix partisan en faveur du PS. La Wallonie a élu lors de l'élection régionale de 2009 des députés régionaux et communautaires provenant de seulement 4 partis (PS, MR, CdH et Ecolo) contre 5 lors de la précédente ${ }^{20}$. Les partis non représentés au Parlement Régional et de la Communauté Française représentent dans les bureaux de dépouillement étudiés entre 5 et $13 \%$ des votes exprimés. Mais dans 5 des 6 cantons, ces partis représentent entre 16 et $33 \%$ des partis cités parmi les bulletins annulés suite à une erreur de vote.

Il semble donc qu'une partie des électeurs blancs et nuls sont en réalité des personnes ayant effectué un choix partisan, bien que contraire à la règle électorale, en faveur de partis marginaux essentiellement protestataires. 
Tableau 4. Nombre et proportion des erreurs de vote parmi le vote blanc et nul (par bureaux de dépouillement - Régionales et Européennes 2009).

\begin{tabular}{|l|l|l|l|}
\hline Cantons & Nb d'erreurs & $\begin{array}{l}\text { Nb de votes } \\
\text { blancs et nuls }\end{array}$ & \% d'erreurs \\
\hline Arlon & 55 & 279 & 19,71 \\
Ath & 8 & 84 & 9,52 \\
Namur & 147 & 716 & 20,53 \\
Stavelot & 18 & 190 & 9,47 \\
Virton & 12 & 139 & 8,63 \\
Walcourt & 79 & 614 & 12,87 \\
TOTAL & 319 & 2022 & 15,78 \\
\hline \multicolumn{2}{|l}{} \\
\hline \multicolumn{2}{|l}{ Source : base de données personnelle, Ministère de l'Intérieur 2009 } \\
\hline
\end{tabular}

\section{Conclusions}

L'intérêt de notre article a donc été de traiter d'un comportement de vote relativement oublié des analyses scientifiques grâce à des données à la fois écologiques et individuelles. Cette approche à deux échelles nous a permis d'éclairer les motivations individuelles de l'électeur "blanc et nul" et de comprendre au niveau agrégé la spatialité de ce vote.

Il apparaît ainsi que le vote blanc et nul peut être au niveau écologique expliqué par trois phénomènes. Tout d'abord, on a constaté un effet de frontière: le vote obligatoire poussant semble-t-il les Belges fortement marqués par la France ou le Luxembourg à voter blanc et nul. Ensuite, le modèle a mis en exergue l'influence du type de vote. Le vote manuel étant le seul à permettre les erreurs de vote, le vote blanc et nul est moindre dans les cantons de vote électronique. On pourrait donc calculer le vote blanc et nul théorique des cantons votant électroniquement en rajoutant la part d'erreurs de vote constatée en moyenne. Nous atteindrions probablement en zone urbaine des scores proches de ceux observés à Namur. Enfin, la ruralité contribue aussi à expliquer la spatialité du vote blanc et nul, bien que cette variable soit plus explicative dans les années 1990 que maintenant. Cela confirme la tendance à l'augmentation de la part des votes blancs et nuls en zone urbaine et la diminution relative de ce que la littérature nomme l'abstention cachée en milieu rural.

Par ailleurs, les modèles nous ont permis de montrer que le niveau socio-économique est significativement lié au vote blanc et nul en 2009, alors qu'il ne l'était pas du tout en 1991, ce qui tend à confirmer le constat sur "l'urbanisation du vote blanc et nul", d'autant plus qu'au niveau intra-urbain, nous avons montré la proximité des géographies du vote blanc et nul et de déterminants sociaux relatifs aux populations en difficulté (Pion, 2009).

Notre étude individuelle sur une base de données originale et inédite en Belgique a quant à elle permis de catégoriser les différents types de bulletins qui ont été annulés aux élections régionales et européennes du 7 juin 2009 en Wallonie et de donner un sens à une 
partie d'entre eux. Ainsi, en moyenne, $35 \%$ des votes annulés sont en réalité blancs, vierges de toute trace de vote. Cette proportion est plus prononcée en milieu rural qu'en milieu urbain et atteint près de la moitié des votes annulés dans les zones rurales frontalières de la France. Le reste, non blanc, se répartit en trois grands types: les bulletins rayés et griffonnés, les bulletins annotés et ceux où une erreur de vote a manifestement été commise.

Les bulletins rayés représentent environ $35 \%$ des votes blancs et nuls, soit autant que les votes blancs. Tout comme ces derniers, ils restent mystérieux quant au sens à leur donner.

Les bulletins annotés, soit $15 \%$ des votes annulés en moyenne, permettent cette fois de mettre des mots sur les motivations des électeurs ayant vu leur bulletin annulé pour cause d'écritures. Ce mode d'expression est plus fréquent dans les cantons urbains comme à Namur ou Arlon. L'analyse des mots, injonctions et phrases écrites sur les bulletins récupérés nous permet de séparer les motivations à la base de ces votes en trois : la protestation vis-à-vis du système politique, l'indifférence et le refus de choisir.

La dernière catégorie de votes annulés regroupe les bulletins marquant une erreur de vote, soit $15 \%$ des votes blancs et nuls. Le choix de candidats dans plusieurs listes est la raison principale de ces annulations et résulte de l'incompétence et de la méconnaissance de la règle électorale de la part des électeurs. Ce type d'annulation représente près du quart des votes blancs et nuls dans les cantons urbains contre moins de $10 \%$ dans les cantons ruraux de Ath et Virton. Nous avons par ailleurs montré que les choix partisans effectués sur ces bulletins annulés diffèrent de ceux validés. Alors que les votes pour des partis non représentés au Parlement Wallon dans les bureaux de dépouillement étudiés représentent moins de $10 \%$ des votes effectifs en moyenne, $25 \%$ des bulletins annulés malgré un choix partisan choisissent un parti marginal.

En combinant les deux analyses, écologique et individuelle, on peut donc relever deux types de votes blanc et nul, à la fois dans la forme (type de vote blanc et nul émis) et dans le fond (sens à donner à ce vote).

51 En milieu urbain, le vote blanc et nul prend plus fréquemment la forme d'annotations, où l'on perçoit le désintérêt pour la politique et la colère vis-à-vis des élites politiques, ou encore des erreurs de vote manifestes. Emanant semble-t-il des classes populaires faiblement éduquées ou de primo-votants peu au fait de la règle électorale, ces votes blancs et nuls urbains sont en augmentation.

52 En milieu rural, le vote blanc et nul reste largement ininterprétable par les bulletins de vote étant donné que plus des trois quarts sont vierges ou rayés sans plus d'indication. Il semble donc bien qu'ils représentent soit une abstention cachée soit le fruit d'une méconnaissance ou d'un désintérêt pour le jeu partisan belge, émanant notamment d'électeurs tournés vers les pays frontaliers par des relations familiales ou de travail. Le vote blanc et nul est par ailleurs beaucoup moins important dans les communes frontalières aux élections communales qu'aux législatives. Cela signifie probablement que les Belges tournés vers l'étranger peuvent se sentir concernés par les élections communales mais pas par les nationales. Par contre, dans les communes rurales peu marquées par l'étranger, le vote blanc et nul fait véritablement office d'abstention cachée, aussi bien aux élections communales qu'aux autres élections. 


\section{BIBLIOGRAPHIE}

BON F., CHEYLAN J.P. (1988), La France qui vote, Paris, Poche.

BUSSI M. (1998), Éléments de Géographie électorale à travers l'exemple de la France de l'Ouest, Rouen, PUR.

BUSSI M., COLANGE C., GOSSET J.P. \& FOURQUET J. (2004), “Elections régionales 2004 : jeux d'échelles entre local, régional et national. L'enseignement des cartes de France par canton", Cybergeo, mis en ligne le 25 mars 2004.

DELOYE Y. \& IHL O. (1991), "Des voix pas comme les autres. Votes blancs et votes nuls aux élections législatives de 1881”, Revue française de sciences politiques, 41, 2, pp. 141-170.

DELRUELLE-VOSSWINKEL N., NOËL F., VANLAER F. \& VANDERMOTTEN C., (1989), "Les élections communales du 9 octobre 1988 : évolution des familles politiques et géographie électorale", Bulletin trimestriel du Crédit communal de Belgique, $\mathrm{n}^{\circ}$ 169, pp. 31-50.

DELWIT P. (1992), "L'abstentionnisme politique en Europe et le cas belge”, Cahiers Marxistes, $\mathrm{n}$ ० 183, pp. 73-85.

DE WINTER L. \& ACKAERT J. (1994), "Le non-vote en Wallonie", in FROGNIER A.P., AISH VAN VAERENBERGH A.M., Élections : la fêlure? Enquête sur le comportement des Wallons et des francophones, Bruxelles, De Boeck Université.

DE WINTER L., DUMONT P., ACKAERT J. (2003), "La participation électorale réelle et potentielle : des vertus du vote obligatoire”, in FROGNIER A.P. \& AISH A.M., Élections la rupture? Le comportement des belges face aux élections de 1999, Bruxelles, De Boeck, pp. 54-69.

DURAND O. (1999), Le vote blanc : pour un suffrage vraiment universel, Paris, L'Harmattan.

GUERIN-PACE F. (1997), “La statistique textuelle. Un outil exploratoire en sciences sociales”, Population, 52, 4, pp. 865-887.

MARISSAL P., MEDINA LOCKHART P., VAN HAMME G. \& VANDERMOTTEN C. (2007), Atlas de Belgique, Géographie politique, Bruxelles, PSF.

MCALLISTER I. \& MAKKAI T. (1993), "Institutions, society or protest ? Explaining invalid votes in Australian elections", Electoral Studies, 12, pp. 23-40.

PION G. (2009), “Géographie des comportements de vote protestataires en Wallonie : une approche infra-communale à Charleroi et Mons”, Belgeo, 2, pp. 153-168.

PION G. (2010), “Le comportement électoral des Italiens de Belgique : une spécificité ?”, Colloque sur les Italiens de Belgique, Bruxelles, 18 octobre 2010.

PION G. \& VAN HAMME G. (2011), “Les destins divergents des régionalismes flamands et francophones : une perspective historique", Echogeo, à paraître.

POWER T.J. \& ROBERTS J.T. (1995), “Compulsory Voting, Invalid Ballots, and Abstention in Brazil”, Political Research Quarterly, 48, 4, pp. 795-826.

STEIFBOLD R.P. (1965), “The signifiance of void ballots in West German elections”, American Political Science Review, 59, pp. 391-407. 
UGGLA F. (2008), "Incompetence, alienation, or calculation ? : Explaining levels of invalid ballots and extra-parliamentary votes", Comparative Political Studies, 41, 8, pp. 1141-1164.

VANDERMOTTEN C. (1992), “Géographie de l'abstention et du vote blanc et nul aux législatives de novembre 1991”, Cahiers Marxistes, n 183, pp. 86-89.

ZULFIKARPASIC A. (2001), "Le vote blanc : abstention civique ou expression politique”, Revue française de sciences politiques, 51, 1-2, pp. 247-268.

\section{NOTES}

1. Le terme "non valable" est aussi employé par certains pour désigner les votes n'entrant pas en ligne de compte pour la répartition des élus rapportés aux votes exprimés, et non pas aux inscrits. Toutes ces dénominations entraînent une confusion légitime.

2. La Région bruxelloise, la province d'Anvers, l'arrondissement de Liège, la Communauté germanophone et une trentaine d'autres cantons situés principalement en Flandre.

3. On notera cependant l'exception de l'Italie.

4. Parfois même on peut déclarer avoir voté soit blanc soit nul.

5. Pour l'analyse en composantes principales tentant d'établir un niveau socio-économique des cantons wallons.

6. On travaillera pour ce faire sur une variable binaire, 0 pour le vote manuel et 1 pour le vote électronique.

7. On a travaillé ici sur les enquêtes socio-économiques de 1991 et 2001 . Le facteur 1 explique ici $51,2 \%$ de l'inertie totale en 1991 et $46,9 \%$ en 2001 et oppose nettement pour les deux dates les territoires comprenant une forte proportion de pensionnés, de chômeurs et d'isolés à ceux dans lesquels se retrouve une proportion importante d'actifs, de propriétaires, de logements récents, de couples avec enfants ainsi qu'un haut revenu médian.

8. Nous n'avons ici tenu compte que des naturalisés récents. On a calculé la proportion d'étrangers naturalisés belges dans les deux années précédant les scrutins de 1991 et 2007. Cette proportion varie entre 0 et $1,2 \%$ en 1991 et 0 et $3 \%$ en 2007. Au contraire des naturalisations, la part de jeunes atteignant l'âge de 18 ans varie très peu selon les cantons (entre 2,2 et $3 \%$ ). Nous avons donc exclu cette variable de l'analyse.

9. Un conjoint belge, l'autre soit français, soit luxembourgeois, soit allemand.

10. On a donc tenu compte de la période 1989-1991 pour les élections fédérales de 1991, de la période 2005-2007 faute de mieux pour les Européennes 2009, et du nombre total d'étrangers naturalisés sur la période 1991-2007 rapporté à la population électorale moyenne du canton pour le champ de force. Notons que si cette dernière variable semble un peu s'écarter des deux autres, le coefficients de corrélation entre les trois sont supérieurs à 0,85 .

11. En 1991, on utilisera la part des frontaliers dans la population cantonale faute de mieux.

12. Nous excluons de l'analyse les cantons de la Communauté germanophone, Eupen et Saint Vith, ainsi que celui de Aubel où les électeurs des Fourons peuvent voter, ce qui ramène à 94 les cantons étudiés.

13. Le fond de vote est le score minimum d'un courant électoral sur une période donnée, le plafond de vote est le score maximum.

14. Sauf dans la première régression, le vote électronique n'étant d'application dans certains cantons qu'à partir de 1994.

15. Il faut noter la plus grande colinéarité entre degré d'urbanité et primo-votants en 2001 qu'en 1991 qui explique en partie cette évolution.

16. On peut cependant arguer que cette hypothèse de notre part est partiellement valable dans le cas des primo-votants. 
17. Le match de barrage afin d'attribuer le titre de champion de Belgique de football entre le Standard de Liège et le RSCA Anderlecht a cristallisé l'attention médiatique lors de la fin du mois de mai 2009. On a comptabilisé $2,8 \%$ des injonctions relatives à cet événement.

18. Les éventuelles fautes d'orthographe ont été laissées comme telles dans nos citations.

19. Avec plus ou moins de souplesse selon les bureaux de dépouillement...

20. Le FN a perdu ses 3 députés régionaux lors de sa débâcle électorale du 7 juin 2009.

\section{RÉSUMÉS}

Le vote blanc et nul est probablement le comportement de vote le plus laissé pour compte dans les études électorales. Pourtant dans une démocratie comme la Belgique, ce vote n'est pas négligeable et présente une géographie atypique mettant en exergue les régions frontalières. Nous analyserons donc dans cet article ce comportement de vote méconnu de manière à la fois écologique et individuelle. Après avoir bâti un modèle de régression tentant de rendre compte de la variation spatiale du vote blanc et nul en Wallonie grâce à des variables démographiques et socio-économiques, nous étudierons les informations issues de près de 2000 bulletins de votes blancs et nuls récupérés dans des services d'archives électorales. L'étude mettra ainsi en lumière l'opposition entre votes blancs et nuls ruraux et urbains. Les premiers, très nombreux notamment le long des frontières nationales, revêtent le caractère soit d'une abstention cachée soit d'un désintérêt pour la politique belge de la part d'électeurs tournés vers les pays frontaliers. Les seconds sont quant à eux la combinaison d'un rejet de la politique et d'une méconnaissance des règles électorales par les classes populaires et les primo-votants.

Blank and null votes are probably the electoral behaviour least taken into account by electoral studies. Though, in a democracy like Belgium, these votes are far from negligible and offer an untypical geography which underlines border areas. This paper will analyze this unknown electoral behaviour in an ecological and individual way. We will first build a multiple regression model in order to try to debrief the spatial variation of blank and null votes in Wallonia thanks to demographic and socioeconomic variables. Secondly, we will study the information retained by more than 2000 spoiled ballot papers. The study will highlight the opposition between rural and urban blank and null votes. The first ones are numerous, notably along the national borders, and reflect essentially hidden abstention or lack of interest in Belgian political life by citizens turned, in their private or professional life, towards France or Luxembourg. The second combine rejection of politics and ignorance of electoral rules in popular classes and first time voters.

\section{INDEX}

Mots-clés : blanc et nul, Wallonie, frontières, bulletins de vote, géographie électorale

Keywords : blank and null votes, Wallonia, borders, ballot papers, electoral geography 
AUTEUR

\section{GEOFFREY PION}

Université Libre de Bruxelles - IGEAT, geofpion@ulb.ac.be 